\title{
Interactions between CD44 and hyaluronan in leukocyte trafficking
}

\author{
Braedon McDonald ${ }^{1,2}$ and Paul Kubes ${ }^{2 *}$ \\ 1 Department of Medicine, University of British Columbia, Vancouver, BC, Canada \\ ${ }^{2}$ Snyder Institute for Chronic Diseases, University of Calgary, Calgary, AB, Canada
}

\section{Edited by:}

David Naor, Hebrew University of Jerusalem, Israel

Reviewed by:

Giuseppe Maurizio Campo, University of Messina, Italy

Carol A. De La Motte, The Cleveland

Clinic, USA

\section{*Correspondence:}

Paul Kubes, Department of

Physiology and Pharmacology, Snyder Institute for Chronic Diseases,

University of Calgary, 3330 Hospital

Drive NW, Calgary, AB T2N 4N1,

Canada

e-mail:pkubes@ucalgary.ca
Recruitment of leukocytes from the bloodstream to inflamed tissues requires a carefully regulated cascade of binding interactions between adhesion molecules on leukocytes and endothelial cells. Adhesive interactions between CD44 and hyaluronan (HA) have been implicated in the regulation of immune cell trafficking within various tissues. In this review, the biology of CD44-HA interactions in cell trafficking is summarized, with special attention to neutrophil recruitment within the liver microcirculation. We describe the molecular mechanisms that regulate adhesion between neutrophil CD44 and endothelial HA, including recent evidence implicating serum-derived hyaluronan-associated protein as an important co-factor in the binding of HA to CD44 under flow conditions. CD44-HA-mediated neutrophil recruitment has been shown to contribute to innate immune responses to invading microbes, as well as to the pathogenesis of many inflammatory diseases, including various liver pathologies. As a result, blockade of neutrophil recruitment by targeting CD44-HA interactions has proven beneficial as an anti-inflammatory treatment strategy in a number of animal models of inflammatory diseases.

Keywords: inflammation, leukocyte recruitment, leukocyte trafficking, CD44, hyaluronan

\section{INTRODUCTION}

The transit of leukocytes from the bloodstream into inflamed tissues involves a series of sequential events, each mediated by the engagement of adhesion molecules on leukocytes and their ligands on vascular endothelium. First, cells flowing in the bloodstream tether to the vessel wall, and begin rolling along the luminal surface. Recent advances in live-cell imaging have revealed that rolling cells form surface appendages called "long tethers" and "slings" that stabilize the rolling phase to enable this dynamic cell-cell interaction to occur at high shear stress $(1,2)$. Subsequently, cells firmly adhere to the vascular endothelium, and begin crawling along the vessel wall until they arrive at a favorable site for transendothelial migration out of the vasculature (3). Upon arrival within the inflamed tissue, leukocytes are guided by gradients of chemoattractants to shepherd them toward their final target where they carry out their effector functions (4).

Each phase of the multi-step cascade of leukocyte recruitment involves binding interactions between adhesion molecules on leukocytes and their ligands on endothelium. Selectins and selectin ligands are the prototypic adhesion molecules that mediate tethering and rolling, through their ability to form transient adhesive "catch" bonds at high shear stress (5). Firm adhesion is classically ascribed to binding between integrins on immune cells (Mac-1, LFA-1, VLA-4, etc.) and receptors of the immunoglobulinsuperfamily on endothelium (ICAM-1, VCAM-1, etc.). The ability of leukocytes such as neutrophils to locomote across the luminal surface of blood vessels via intra-luminal crawling has also been shown to involve integrins, while transmigration requires the coordinated interplay of multiple molecular binding partners (6).

An explosion of research in the field of leukocyte trafficking in the past 25 years has identified many additional "non-classical" adhesion molecules that can support cell recruitment in the myriad of cell, organ, and disease-specific contexts that are required for a functional immune system (7). Among these, CD44 and its ligand, hyaluronan (HA), have emerged as important adhesion molecules required for cell trafficking in multiple organs, and contribute to the pathogenesis of a variety of inflammatory diseases. CD44 is a type I transmembrane glycoprotein expressed by multiple hematopoietic and non-hematopoietic cells. CD44 is encoded by a single gene, but can be expressed in over 20 isoforms as a result of alternative splicing, and can undergo an array of post-translational modifications, enabling nuanced regulation and diverse functions. The key ligand for CD44, $\mathrm{HA}$, is a member of the glycosaminoglycan family of extracellular matrix molecules, formed from a basic structural unit of repeating dissacharides of $\mathrm{N}$-acetylglucosamine and $\mathrm{N}$-glucuronic acid. HA is a nearly ubiquitous component of the extracellular matrix, and can be produced by multiple cell types, including endothelium [for a comprehensive review of the regulation of HA synthesis and metabolism, see Ref. (8)]. Below, we review the biology of CD44-HA interactions in immune cell trafficking, explore the molecular mechanisms that regulate the binding between these molecules, and highlight the critical role of CD44-HA-mediated leukocyte recruitment in the pathogenesis of inflammatory diseases.

\section{CD44 AND HYALURONAN IN THE LEUKOCYTE RECRUITMENT CASCADE \\ ROLLING}

Seminal experiments using a parallel-plate flow-chamber system to simulate the hemodynamic environment of post-capillary venules were the first to demonstrate a role for CD44 and HA in leukocyte trafficking. Lymphocytes were observed to roll on 
HA-coated plates, and this rolling could be inhibited by incubating the lymphocytes with a function-blocking antibody against CD44 (9). Subsequently, similar in vitro systems were used to demonstrate that CD44 on lymphocytes could support rolling on a monolayer of cultured endothelial cells in an HA-dependent manner (9, 10). These findings were confirmed in vivo in a model of peritonitis, in which T-cells utilized CD44-HA interactions to home to the inflamed peritoneal cavity (10). Recruitment of superantigenactivated T-cells into the inflamed peritoneum could be inhibited by CD44-blocking antibodies, as well as intravenous infusion of hyaluronidase to degrade endothelial HA (10). However, unlike lymphocytes, neutrophils are unable to roll on a purified HA substrate in vitro, and neutrophil rolling on cultured endothelial cells as well as the endothelium of post-capillary venules in vivo is not dependent on CD44-HA interactions.

In addition, CD44 can also contribute to leukocyte rolling through interactions with non-HA ligands. Teder and colleagues identified CD44 as a novel E-selectin ligand, and showed that binding between these molecules can support neutrophil rolling in mice and humans (11). Specifically, engagement between neutrophil CD44 and endothelial E-selectin was required for slow rolling, whereas P-selectin-PSGL-1 interactions were required for initial tethering and fast rolling. Furthermore, neutrophil CD44 from patients with leukocyte adhesion deficiency (LAD) syndrome type II (due to mutations of the GDP-fucose transporter gene, resulting in complete deficiency of fucosylated selectin-ligand moieties) was unable to bind E-selectin, implicating CD44-Eselectin interactions in the pathogenesis of this disease (11). In addition to neutrophils, T-lymphocytes also utilize CD44-Eselectin interactions for slow rolling in vivo, in a manner that is independent of the contribution of CD44-HA interactions (12).

\section{FIRM ADHESION}

Intravital microscopy of the inflamed cremaster muscle of mice has revealed that unlike rolling, firm adhesion of neutrophils can be supported by interactions between CD44 and HA. Blocking antibodies against CD44, genetic deletion of CD44, and removal of HA from the vascular endothelium have each been shown to attenuate neutrophil adhesion (but not rolling), within post-capillary venules (13). Similarly, as described below, CD44 can support neutrophil adhesion within low-shear microvascular beds such as the liver sinusoids, in which neutrophils undergo primary adhesion without a pre-requisite rolling step (14).

A role for CD44 in mediating firm adhesion of lymphocytes has been suggested, but the exact functional contribution of CD44 is less clear. Both in vitro and in vivo, disruption of CD44HA interactions limits the adhesion of activated lymphocytes to endothelium $(10,15)$. However, given that CD44-HA binding also mediates the pre-requisite rolling step, the reduction of firm adhesion may be a secondary effect. This question has been addressed directly by Nandi and colleagues, who investigated the contribution of CD44 versus integrins (the prototypic molecules supporting firm adhesion) to adhesion of activated T-cells to endothelium under flow conditions (16). Interestingly, it was found that CD44 was required for firm adhesion, although not through binding interactions with HA. Co-immunoprecipitation experiments revealed bi-molecular complex formation between
CD44 (which supports rolling) and the integrin VLA-4 (which supports adhesion) (16). Expression of truncated forms of CD44 that lacked the cytoplasmic tail prevented physical coupling of CD44 with VLA-4, resulting in cells that could roll (using CD44) but were unable to adhere. These experiments revealed that while CD44-HA interactions support lymphocyte rolling, CD44 is also required for adhesion by directly collaborating with integrins to enable the formation of high affinity binding to VCAM-1.

\section{TRANS-ENDOTHELIAL MIGRATION AND CHEMOTAXIS}

Conflicting data exist regarding the function of CD44 and HA in trans-endothelial migration and chemotaxis through interstitial tissues. With respect to transmigration, neutrophil transit across epithelial monolayers in vitro was found to be attenuated when neutrophil CD44 was activated, but was unaffected by functional blockade of the HA-binding domain (17). Importantly, these experiments tested neutrophil migration across intestinal epithelial monolayers under static conditions, and therefore their applicability to trans-endothelial migration under flow is unknown. In contrast, visualization of neutrophil recruitment in vivo within the inflamed cremaster muscle of mice demonstrated reduced transmigration in CD44 knockout animals (13). The molecular mechanism by which CD44 contributes to trans-endothelial migration has not been studied. Similarly, the role of CD44-HA interaction in chemotaxis is incompletely understood. Khan et al. found conflicting results between the ability of CD44-deficient neutrophils to migrate toward chemoattractants in vivo versus in vitro (13). In an under-agarose migration assay, neutrophils from CD44-deficient mice had markedly impaired migration toward MIP-2/CXCL2 compared to wild-type neutrophils. However, neutrophil chemotaxis within cremaster muscle in vivo was independent of CD44-HA interactions. The differences observed between in vitro and in vivo conditions is similar to that observed for the role of integrins in neutrophil chemotaxis, and may be the result of differences in the matrix composition through which cells must migrate in the various models $(18,19)$. Overall, these studies reveal a paucity of data on the contribution of CD44-HA interactions to the final phases of leukocyte recruitment, and highlight a need for further research in this area.

\section{CD44-HA-MEDIATED LEUKOCYTE RECRUITMENT IN INFLAMMATORY DISEASE}

Numerous studies have confirmed the importance of CD44 and HA in leukocyte recruitment within a variety of organs in vivo. Antibody blockade of CD44, CD44 deficiency, or enzymatic depletion of endothelial HA (by administration of hyaluronidase) have been shown to decrease neutrophil, monocyte, and/or lymphocyte recruitment and attenuate disease activity in models of arthritis $(20,21)$, dermatitis (22), peritonitis (10), myositis (13), experimental autoimmune encephalomyelitis (23), orchitis (24), retinitis (25), allergic asthma (26), and graft-versus-host disease (27). However, perhaps one of the best-characterized roles for CD44HA interactions in pathological leukocyte recruitment is seen in inflammatory liver disease.

The liver vasculature represents a unique structural and hemodynamic environment for leukocyte recruitment, as the majority of infiltrating cells are recruited within the dense labyrinth of 
low-flow, low-shear sinusoids. As a result, the mechanisms of leukocyte trafficking within these vessels deviate from the classic multi-step paradigm, in that recruitment does not require a rolling phase (28). Instead, cells are observed to undergo primary adhesion within these low-flow vessels. Therefore, the pleiotropic abilities of CD44-HA interactions to support all steps from tethering to firm adhesion make this binding interaction well suited to the biology of the liver sinusoids. Furthermore, multiple studies using a variety of imaging techniques have demonstrated that the luminal surface of liver sinusoidal endothelium is densely coated with HA $(14,29,30)$. Unlike other vascular beds, in which endothelial cell CD44 is required to anchor HA to the vessel wall, liver sinusoidal endothelial cells (LSEC) do not use CD44 to anchor HA, but instead express a variety of scavenger receptors that capture circulating HA on the cell surface, and present it to passing leukocytes before finally promoting its endocytosis and clearance from the bloodstream (31-33). However, no studies to date have investigated the functional role of these scavenger receptors in leukocyte adhesion to HA within liver sinusoids.

A prominent role for CD44-HA interactions in immune cell recruitment to the liver was first observed in a model of acute hepatic inflammation due to sepsis/endotoxemia. Direct visualization of neutrophil trafficking within liver sinusoids revealed that blockade of CD44-HA interactions led to a 50-70\% reduction in the number of adherent neutrophils (14). While CD44 can engage with other ligands to support leukocyte-endothelial interactions within other vascular beds (E-selectin and VLA-4, as described above), this promiscuous activity is not observed in liver sinusoids, as multiple studies have confirmed that selectins and $\alpha 4$-integrins do not contribute to neutrophil recruitment within the liver $(14,28,34)$. Menezes and colleagues performed a more in-depth functional analysis of CD44-HA interaction using confocal intravital microscopy in mouse models of endotoxemia and Gram-negative bacterial sepsis (35). It was found that in addition to mediating initial adhesion, CD44 was also required for subsequent stages of recruitment including cell spreading and the initiation of intravascular crawling.

Subsequently, the role of CD44-HA interactions in cell recruitment to the liver has been observed for other cell types in a variety of inflammatory contexts. First, Shi et al. revealed that monocyte recruitment to foci of Listeria monocytogenes infection in the liver was reduced by more than $50 \%$ by anti-CD 44 antibody treatment (36). Interestingly, this study observed a roughly equivalent reduction in monocyte infiltration when Mac-1-ICAM-1 interactions were blocked, but it is not known how each receptorligand pair contributes to cell recruitment. One possibility is that CD44 and Mac-1 function cooperatively to promote initial monocyte adhesion. Alternatively, these molecules may function in a sequential manner, with CD44 mediating initial tethering and adhesion, while Mac-1 supports subsequent intravascular crawling toward the bacterial targets. Similar to monocytes and neutrophils, an important role for CD44-HA interactions has also been observed in cytotoxic T-lymphocyte (CTL) recruitment to the inflamed liver in a mouse model of viral hepatitis (37). Hepatitis B transgenic mice develop substantial lymphocytic inflammatory infiltrates within the liver, whereas transgenic mice that are also CD44 deficient have markedly attenuated CTL recruitment. Interestingly, adoptive transfer experiments revealed that CD44 was required on endothelium, but not on CTLs. This stands in stark contrast to the role of CD44 in neutrophil and monocyte recruitment, in which CD44 is required on leukocytes but not endothelium $(14,36)$. The mechanism underlying this observation is not known, but possible explanations include interactions with non-HA ligands on CTLs, or possibly a role for CD44 in upstream inflammatory signaling rather than as an adhesion molecule in CTL trafficking.

A number of recent studies have described a critical role for CD44-HA interactions in the pathogenesis of fatty-liver disease, the leading cause of chronic liver disease in United States and a growing problem worldwide (38). CD44-deficient mice have markedly attenuated hepatitis in a mouse model of non-alcoholic steatohepatitis (NASH), induced by administration of a lithogenic diet $(39,40)$. Leukocytes harvested from lithogenic diet-fed mice display significant upregulation of their HA-binding capacity (39). This correlated with diminished leukocyte infiltration into the livers of CD44-deficient animals. Using a similar model of lithogenic diet-induced hepatic steatosis, Kang et al. observed that CD44-deficient mice displayed reduced leukocyte infiltration into the liver, in addition to broader defects in steatosis development, adipose tissue inflammation, and insulin resistance (40). Therefore, in addition to supporting leukocyte infiltration into the liver and the development of NASH, CD44 may be a keystone to the systemic pathogenesis of obesity-associated diseases and the metabolic syndrome.

Lastly, CD44-HA interactions have been implicated in the trafficking of hematopoietic stem cells (HSC) to the liver. In vitro experiments studying adhesive interactions between HSC and cultured LSEC found that binding was partially dependent on CD44HA interactions (41). Similarly, CD44 blockade reduced adhesion of HSC to frozen liver sections from patients with primary biliary cirrhosis and alcoholic liver disease (41). While these findings await confirmation in vivo, a similar role for CD44-HA interactions has been observed in mesenchymal stem cell recruitment to the kidney in an animal model of acute tubular necrosis (42). Together, these findings suggest that CD44-HA interaction are important not only for the generation of inflammatory responses, but may also be critical to the resolution phase and tissue regeneration by facilitating stem cell homing to injured tissues. This would add an additional role to the growing list of contributions made by CD44 to the resolution of inflammatory responses (43).

\section{REGULATION OF CD44-HA INTERACTIONS IN CELL TRAFFICKING}

Despite the fact that CD44 is constitutively expressed on most leukocytes, and HA is a ubiquitous component of the extracellular matrix, trafficking leukocytes only adhere to HA under inflammatory conditions (44). Engagement between CD44 and HA can be regulated at a number of levels including the quantity of surface expression, as well as their functional states of activation.

\section{REGULATION OF CD44 FUNCTION}

CD44 expression on the surface of leukocytes can be upregulated in response to a variety of stimuli including antigen receptor 
cross-linking, mitogens, cytokines, chemokines, and bacterial products (44). Numerous studies have demonstrated that upregulation of CD44 expression can lead to augmented recruitment of various leukocyte subsets to sites of inflammation. Conversely, defects that result in impaired CD44 surface expression can result in attenuation of leukocyte trafficking kinetics. For example, neutrophils from Rab27a-deficient mice display reduced surface expression of CD44, and as such were found to have impaired adhesion within liver sinusoids in response to endotoxemia (45). Although the precise pathway through which Rab27a controls CD44 expression is not fully elucidated, in vitro observations suggest that this molecule may regulate the intracellular trafficking and surface presentation of CD44 on the plasma membrane within neutrophils. Interestingly, genetic defects in the Rab27a gene in humans results in a rare immunodeficiency syndrome (Griscelli syndrome 2) characterized by defective neutrophil, NK cell, and CTL function (46). Given the defects in CD44 expression on neutrophils observed in Rab27a-deficient mice, it will be of interest in future studies to determine if the impairments in CTLs and NK cells are also linked to defective CD44 expression and function.

It is not only the absolute quantity of CD44 expression on the cell surface that regulates binding to HA but also the relative expression compared to other adhesion molecules. Studies of neutrophil adhesion in the liver have revealed that the balance between CD44 and integrin (Mac-1) expression can dramatically alter the role of CD44 in leukocyte recruitment. Menezes and colleagues found that CD44-HA interactions are the dominant mechanisms of adhesion in response to endotoxemia/sepsis, whereas CD44 is dispensable when hepatic inflammation was induced by a single neutrophil chemoattractant (fMLP), wherein adhesion required Mac-1 (35). Analysis of surface expression levels revealed equivalent quantities of CD44 expression regardless of the stimulus, whereas Mac-1 expression was significantly reduced during endotoxemia as a consequence of high circulating IL-10. The authors postulated that during endotoxemia, downregulation of Mac-1 allows for $\mathrm{CD} 44$ to dominate, resulting in preferential adhesion via CD44-HA interactions, highlighting the functional importance of relative CD44 expression levels in the control of neutrophil trafficking.

In addition to regulation of surface expression levels, activation of leukocytes can also induce a multitude of post-translational modifications (phosphorylation, glycosylation, sulfation, and others) that yield a structurally activated, HA-avid form of CD44 [reviewed by Puré and Cuff (44)]. The functional significance of such post-translational modifications has been demonstrated in vitro using flow-chamber experiments, in which lymphocyte activation, and the resultant modifications of $\mathrm{CD} 44$, are required to enable rolling and adhesion upon HA-coated coverslips (47). Similarly, activated lymphocytes that are recovered from inflamed tissues in vivo posses functionally activated forms of CD44, and demonstrate enhanced binding to HA compared to naïve lymphocytes $(10,15)$. Perhaps the most direct evidence for conformational activation of CD44 in the control of CD44-HA interaction comes from studies using the IRAWB14.4 antibody, which binds CD44 and alters its conformation directly to generate high HA-avidity. As a result, this antibody commonly serves as a positive control in HA-binding experiments $(14,15)$.

\section{REGULATION OF HA FUNCTION}

While expression and activation of leukocyte CD44 are clearly important for the regulation of cell trafficking, there is emerging evidence that leukocyte recruitment is also modulated by changes in HA expression and/or function at the level of vascular endothelium. First, endothelial cells have the ability to augment their surface expression of HA under inflammatory conditions. Using cultured endothelial cell lines as well as primary endothelial cells, Siegelman and colleagues found that stimulation with TNF $\alpha$, IL-1 $\beta$, or LPS resulted in upregulated surface expression of HA (48). Interestingly, this phenotype was only observed in endothelial cells derived from microvascular beds (but not larger vessels) in which the majority of leukocyte recruitment occurs (48). Using a parallel-plate flow-chamber assay, the authors confirmed that CD44-dependent lymphocyte rolling was increased fourfold upon activated endothelial monolayers as a result of upregulated surface expression of HA (48).

In addition to quantitative regulation of HA expression, leukocyte trafficking can also be modulated by functional changes to the structure of HA polymers. First, HA polymer length has received a great deal of attention in recent years as an important rheostatat for the inflammatory response, as a result of its multi-functional inflammatory/anti-inflammatory signaling properties mediated by low versus high molecular weight HA (49). Polymer length may also regulate the function of HA as an adhesion molecule, as evidenced by the fact that digestion with hyaluronidase impairs leukocyte-endothelial interactions. In addition, a growing body of research suggests that a major regulator of HA-CD44 interactions involves structural modification of HA induced by a variety of HA-binding proteins. Serum-derived hyaluronan-associated proteins (SHAPs), is an HA-binding protein that has been shown to modulate leukocyte trafficking through functional alterations of the adhesion properties of HA. Structurally, SHAP is composed of the heavy chains (HC) of inter- $\alpha$-trypsin inhibitor, a circulating proteoglycan consisting of a single chondroitin sulfate molecule bound to bikunin (a serine protease inhibitor) and the aforementioned $\mathrm{HC}(\mathrm{HC} 1, \mathrm{HC} 2, \mathrm{HC} 3)$ (50). At sites of inflammation, I $\alpha$ I HC (SHAP) are transferred onto HA through a trans-esterification reaction catalyzed by tumor necrosis factorstimulated gene 6 (TSG-6), yielding a covalently bound SHAP-HA complex (50). SHAP-HA complexes purified from the synovial fluid of patients with rheumatoid arthritis bind with greater avidity to lymphocyte CD44 than native HA alone (51). Furthermore, lymphocyte rolling and adhesion in vitro upon a substratum of SHAP-HA is increased more than fourfold compared to HA alone (51). Some studies have suggested that SHAP-HA complexes may enable adhesion regardless of the activation state of leukocyte CD44 (52). Systematic inhibition studies have confirmed that adhesion to SHAP-HA still occurs exclusively between HA and CD44, and that the role of SHAP is indirect through functional alterations of HA macromolecular structure (51). Immunofluorescence imaging has revealed that binding of SHAP induces profound architectural changes to the macro-structure of HA polymers, resulting in coalescence of HA polymers into multi-celllength cables (52). This concentration of HA polymers may augment binding to leukocytes by enabling high avidity interactions with leukocyte CD44, potentially providing favorable clustering 
of CD44 within plasma membrane microdomains. Alternatively, formation of SHAP-HA complexes may protect HA from degradation or endocytosis, thereby stabilizing the surface landscape of the vascular endothelium to promote interactions with passing leukocytes.

Serum-derived hyaluronan-associated protein-HA complexes are observed in multiple inflamed organs. High concentrations have been demonstrated in the synovial fluid of patients with rheumatoid arthritis (51), and colon tissue from patients with active inflammatory bowel disease (52). Within the colon of patients with IBD, SHAP-HA complexes were observed primarily around blood vessels, as well as within the hyperplastic muscularis layer (52). Within the liver microvasculature, neutrophil adhesion in response to bacterial LPS is preceded by marked induction of SHAP-HA complex formation on the endothelial surface $(14,53)$. Unlike $\mathrm{HA}$, which is present in liver sinusoids constitutively at high levels, SHAP-HA complexes are only seen after exposure to bacterial products or other stimuli, and CD44-bearing neutrophils adhere within regions that are richly decorated with SHAP-HA complexes $(14,54)$. Experiments in mice that lack the SHAP precursor $(\mathrm{I} \alpha \mathrm{I})$, and are therefore SHAP-deficient, have revealed that neutrophil adhesion is reduced by $\sim 50 \%$ compared to wild-type animals (53). Together, these studies implicate functional "activation" of HA in response to SHAP (and perhaps other HA-binding proteins) in the regulation of leukocyte adhesion. Further studies are needed to clarify the mechanisms responsible for the enhanced avidity of HA for CD44 following binding by SHAP, and whether the macro-structural alteration (such as cable formation) that are seen in vitro also occur within the vasculature in vivo.

\section{CONCLUSION}

As illustrated in this issue of Frontiers, CD44 and HA contribute to a remarkably diverse spectrum of biologic processes. As leukocyte adhesion molecules, CD44 and HA display a variety of functional roles in cell trafficking, and contribute broadly to the initiation, propagation, and resolution of inflammatory responses. Within many domains, CD44 and HA are being investigated as potential therapeutic targets to modulate disease pathology, and leukocyte recruitment is no exception. Through our expanded knowledge of the mechanisms regulating CD44 and HA interactions, and how these influence inflammatory responses, we will gain further insight into potential therapeutic targets to treat inflammatory diseases.

\section{ACKNOWLEDGMENTS}

The authors would like to thank Dr. Mary Dunbar for her critical review of this manuscript.

\section{REFERENCES}

1. Sundd P, Gutierrez E, Koltsova EK, Kuwano Y, Fukuda S, Pospieszalska MK, et al. "Slings" enable neutrophil rolling at high shear. Nature (2012) 488(7411):399-403. doi:10.1038/nature11248

2. Sundd P, Gutierrez E, Pospieszalska MK, Zhang H, Groisman A, Ley K. Quantitative dynamic footprinting microscopy reveals mechanisms of neutrophil rolling. Nat Methods (2010) 7(10):821-4. doi:10.1038/nmeth.1508

3. Phillipson M, Heit B, Colarusso P, Liu L, Ballantyne CM, Kubes P. Intraluminal crawling of neutrophils to emigration sites: a molecularly distinct process from adhesion in the recruitment cascade. J Exp Med (2006) 203(12):2569-75. doi:10.1084/jem.20060925
4. Heit B, Robbins SM, Downey CM, Guan Z, Colarusso P, Miller BJ, et al. PTEN functions to 'prioritize' chemotactic cues and prevent "distraction" in migrating neutrophils. Nat Immunol (2008) 9(7):743-52. doi:10.1038/ni.1623

5. Marshall BT, Long M, Piper JW, Yago T, McEver RP, Zhu C. Direct observation of catch bonds involving cell-adhesion molecules. Nature (2003) 423(6936):190-3. doi:10.1038/nature01605

6. Muller WA. Mechanisms of leukocyte transendothelial migration. Annu Rev Pathol (2011) 6:323-44. doi:10.1146/annurev-pathol-011110-130224

7. Phillipson M, Kubes P. The neutrophil in vascular inflammation. Nat Med (2011) 17(11):1381-90. doi:10.1038/nm.2514

8. Jiang D, Liang J, Noble PW. Hyaluronan in tissue injury and repair. Annu Rev Cell Dev Biol (2007) 23:435-61. doi:10.1146/annurev.cellbio.23.090506.123337

9. DeGrendele HC, Estess P, Picker LJ, Siegelman MH. CD44 and its ligand hyaluronate mediate rolling under physiologic flow: a novel lymphocyteendothelial cell primary adhesion pathway. J Exp Med (1996) 183(3):1119-30. doi:10.1084/jem.183.3.1119

10. DeGrendele HC, Estess P, Siegelman MH. Requirement for CD44 in activated T cell extravasation into an inflammatory site. Science (1997) 278(5338):672-5. doi:10.1126/science.278.5338.672

11. Katayama Y, Hidalgo A, Chang J, Peired A, Frenette PS. CD44 is a physiological E-selectin ligand on neutrophils. J Exp Med (2005) 201(8):1183-9. doi:10.1084/jem.20042014

12. Nácher M, Blázquez AB, Shao B, Matesanz A, Prophete C, Berin MC, et al. Physiological contribution of CD44 as a ligand for E-Selectin during inflammatory T-cell recruitment. Am J Pathol (2011) 178(5):2437-46. doi:10.1016/j.ajpath. 2011.01.039

13. Khan AI, Kerfoot SM, Heit B, Liu L, Andonegui G, Ruffell B, et al. Role of CD44 and hyaluronan in neutrophil recruitment. JImmunol (2004) 173(12):7594-601. doi:10.4049/jimmunol.173.12.7594

14. McDonald B, McAvoy EF, Lam F, Gill V, de la Motte C, Savani RC, et al. Interaction of CD44 and hyaluronan is the dominant mechanism for neutrophil sequestration in inflamed liver sinusoids. J Exp Med (2008) 205(4):915-27. doi:10.1084/jem.20071765

15. Bonder CS, Clark SR, Norman MU, Johnson P, Kubes P. Use of CD44 by CD4+ Th1 and Th2 lymphocytes to roll and adhere. Blood (2006) 107(12):4798-806. doi:10.1182/blood-2005-09-3581

16. Nandi A, Estess P, Siegelman M. Bimolecular complex between rolling and firm adhesion receptors required for cell arrest; CD44 association with VLA-4 in T cell extravasation. Immunity (2004) 20(4):455-65. doi:10.1016/S1074-7613(04) 00077-9

17. Si-Tahar M, Sitaraman S, Shibahara T, Madara JL. Negative regulation of epithelium-neutrophil interactions via activation of CD44. Am J Physiol Cell Physiol (2001) 280(3):C423-32.

18. Heit B, Colarusso P, Kubes P. Fundamentally different roles for LFA-1, Mac-1 and alpha4-integrin in neutrophil chemotaxis. J Cell Sci (2005) 118(Pt 22):5205-20. doi:10.1242/jcs.02632

19. Lämmermann T, Bader BL, Monkley SJ, Worbs T, Wedlich-Söldner R, Hirsch K, et al. Rapid leukocyte migration by integrin-independent flowing and squeezing. Nature (2008) 453(7191):51-5. doi:10.1038/nature06887

20. Sarraj B, Ludányi K, Glant TT, Finnegan A, Mikecz K. Expression of CD44 and $\mathrm{L}$-selectin in the innate immune system is required for severe joint inflammation in the proteoglycan-induced murine model of rheumatoid arthritis. J Immunol (2006) 177(3):1932-40. doi:10.4049/jimmunol.177.3.1932

21. Mikecz K, Brennan FR, Kim JH, Glant TT. Anti-CD44 treatment abrogates tissue oedema and leukocyte infiltration in murine arthritis. Nat Med (1995) 1(6):558-63. doi:10.1038/nm0695-558

22. Gonda A, Gál I, Szántó S, Sarraj B, Glant TT, Hunyadi J, et al. CD44, but not L-selectin, is critically involved in leucocyte migration into the skin in a murine model of allergic dermatitis. Exp Dermatol (2005) 14(9):700-8. doi:10.1111/j.0906-6705.2005.00348.x

23. Brocke S, Piercy C, Steinman L, Weissman IL, Veromaa T. Antibodies to CD44 and integrin alpha4, but not L-selectin, prevent central nervous system inflammation and experimental encephalomyelitis by blocking secondary leukocyte recruitment. Proc Natl Acad Sci USA (1999) 96(12):6896-901. doi:10.1073/pnas. 96.12.6896

24. Guazzone VA, Denduchis B, Lustig L. Involvement of CD44 in leukocyte recruitment to the rat testis in experimental autoimmune orchitis. Reproduction (2005) 129(5):603-9. doi:10.1530/rep.1.00329 
25. Xu H, Manivannan A, Liversidge J, Sharp PF, Forrester JV, Crane IJ. Involvement of CD44 in leukocyte trafficking at the blood-retinal barrier. JLeukoc Biol (2002) 72(6):1133-41.

26. Katoh S, Ishii N, Nobumoto A, Takeshita K, Dai S-Y, Shinonaga R, et al. Galectin-9 inhibits CD44-hyaluronan interaction and suppresses a murine model of allergic asthma. Am J Respir Crit Care Med (2007) 176(1):27-35. doi:10.1164/rccm.200608-1243OC

27. Milinkovic M, Antin JH, Hergrueter CA, Underhill CB, Sackstein R. CD44hyaluronic acid interactions mediate shear-resistant binding of lymphocytes to dermal endothelium in acute cutaneous GVHD. Blood (2004) 103(2):740-2. doi:10.1182/blood-2003-05-1500

28. Wong J, Johnston B, Lee SS, Bullard DC, Smith CW, Beaudet AL, et al. A minimal role for selectins in the recruitment of leukocytes into the inflamed liver microvasculature. J Clin Invest (1997) 99(11):2782-90. doi:10.1172/JCI119468

29. Ichida T, Sugitani S, Satoh T, Matsuda Y, Sugiyama M, Yonekura K, et al. Localization of hyaluronan in human liver sinusoids: a histochemical study using hyaluronan-binding protein. Liver (1996) 16(6):365-71. doi:10.1111/j.16000676.1996.tb00763.x

30. Satoh T, Ichida T, Matsuda Y, Sugiyama M, Yonekura K, Ishikawa T, et al. Interaction between hyaluronan and CD44 in the development of dimethylnitrosamine-induced liver cirrhosis. J Gastroenterol Hepatol (2000) 15(4):402-11. doi:10.1046/j.1440-1746.2000.02164.x

31. Schledzewski K, Géraud C, Arnold B, Wang S, Gröne H-J, Kempf T, et al. Deficiency of liver sinusoidal scavenger receptors stabilin-1 and -2 in mice causes glomerulofibrotic nephropathy via impaired hepatic clearance of noxious blood factors. J Clin Invest (2011) 121(2):703-14. doi:10.1172/JCI44740

32. Zhou B, Weigel JA, Fauss L, Weigel PH. Identification of the hyaluronan receptor for endocytosis (HARE). J Biol Chem (2000) 275(48):37733-41. doi:10.1074/jbc.M003030200

33. Weigel JA, Raymond RC, McGary C, Singh A, Weigel PH. A blocking antibody to the hyaluronan receptor for endocytosis (HARE) inhibits hyaluronan clearance by perfused liver. J Biol Chem (2003) 278(11):9808-12. doi:10.1074/jbc. M211462200

34. Fox-Robichaud A, Kubes P. Molecular mechanisms of tumor necrosis factor alpha-stimulated leukocyte recruitment into the murine hepatic circulation. Hepatology (2000) 31(5):1123-7. doi:10.1053/he.2000.6961

35. Menezes GB, Lee W-Y, Zhou H, Waterhouse CCM, Cara DC, Kubes P. Selective down-regulation of neutrophil Mac-1 in endotoxemic hepatic microcirculation via IL-10. J Immunol (2009) 183(11):7557-68. doi:10.4049/jimmunol.0901786

36. Shi C, Velázquez P, Hohl TM, Leiner I, Dustin ML, Pamer EG. Monocyte trafficking to hepatic sites of bacterial infection is chemokine independent and directed by focal intercellular adhesion molecule-1 expression. JImmunol (2010) 184(11):6266-74. doi:10.4049/jimmunol.0904160

37. Kimura K, Nagaki M, Saio M, Moriwaki H, Kakimi K. Role of CD44 in CTLinduced acute liver injury in hepatitis B virus transgenic mice. J Gastroenterol (2009) 44(3):218-27. doi:10.1007/s00535-008-2300-8

38. Younossi ZM, Stepanova M, Afendy M, Fang Y, Younossi Y, Mir H, et al. Changes in the prevalence of the most common causes of chronic liver diseases in the United States from 1988 to 2008. Clin Gastroenterol Hepatol (2011) 9(6):524.e-30.e. doi:10.1016/j.cgh.2011.03.020

39. Egan CE, Daugherity EK, Rogers AB, Abi Abdallah DS, Denkers EY, Maurer KJ. CCR2 and CD44 promote inflammatory cell recruitment during fatty liver formation in a lithogenic diet fed mouse model. PLoS One (2013) 8(6):e65247. doi:10.1371/journal.pone.0065247

40. Kang HS, Liao G, DeGraff LM, Gerrish K, Bortner CD, Garantziotis S, et al. CD44 plays a critical role in regulating diet-induced adipose inflammation, hepatic steatosis, and insulin resistance. PLoS One (2013) 8(3):e58417. doi:10.1371/journal.pone.0058417

41. Crosby HA, Lalor PF, Ross E, Newsome PN, Adams DH. Adhesion of human haematopoietic (CD34+) stem cells to human liver compartments is integrin and CD44 dependent and modulated by CXCR3 and CXCR4. J Hepatol (2009) 51(4):734-49. doi:10.1016/j.jhep.2009.06.021
42. Herrera MB, Bussolati B, Bruno S, Morando L, Mauriello-Romanazzi G, Sanavio F, et al. Exogenous mesenchymal stem cells localize to the kidney by means of CD44 following acute tubular injury. Kidney Int (2007) 72(4):430-41. doi:10.1038/sj.ki.5002334

43. Teder P, Vandivier RW, Jiang D, Liang J, Cohn L, Puré E, et al. Resolution of lung inflammation by CD44. Science (2002) 296(5565):155-8. doi:10.1126/science. 1069659

44. Puré E, Cuff CA. A crucial role for CD44 in inflammation. Trends Mol Med (2001) 7(5):213-21. doi:10.1016/S1471-4914(01)01963-3

45. Johnson JL, Hong H, Monfregola J, Catz SD. Increased survival and reduced neutrophil infiltration of the liver in Rab27a- but not Munc13-4-deficient mice in lipopolysaccharide-induced systemic inflammation. Infect Immun (2011) 79(9):3607-18. doi:10.1128/IAI.05043-11

46. Klein C, Philippe N, Le Deist F, Fraitag S, Prost C, Durandy A, et al. Partial albinism with immunodeficiency (Griscelli syndrome). J Pediatr (1994) 125(6 Pt 1):886-95. doi:10.1016/S0022-3476(05)82003-7

47. Maiti A, Maki G, Johnson P. TNF-alpha induction of CD44-mediated leukocyte adhesion by sulfation. Science (1998) 282(5390):941-3. doi:10.1126/science.282 . 5390.941

48. Mohamadzadeh M, DeGrendele H, Arizpe H, Estess P, Siegelman M. Proinflammatory stimuli regulate endothelial hyaluronan expression and CD44/HAdependent primary adhesion. J Clin Invest (1998) 101(1):97-108. doi:10.1172/ JCI1604

49. Petrey AC, de la Motte CA. Hyaluronan, a crucial regulator of inflammation. Front Immunol (2014) 5:101. doi:10.3389/fimmu.2014.00101

50. Day AJ, de la Motte CA. Hyaluronan cross-linking: a protective mechanism in inflammation? Trends Immunol (2005) 26(12):637-43. doi:10.1016/j. it.2005.09.009

51. Zhuo L, Kanamori A, Kannagi R, Itano N, Wu J, Hamaguchi M, et al. SHAP potentiates the CD44-mediated leukocyte adhesion to the hyaluronan substratum. J Biol Chem (2006) 281(29):20303-14. doi:10.1074/jbc.M506703200

52. de la Motte CA, Hascall VC, Drazba J, Bandyopadhyay SK, Strong SA. Mononuclear leukocytes bind to specific hyaluronan structures on colon mucosal smooth muscle cells treated with polyinosinic acid:polycytidylic acid: interalpha-trypsin inhibitor is crucial to structure and function. Am J Pathol (2003) 163(1):121-33. doi:10.1016/S0002-9440(10)63636-X

53. McDonald B, Jenne CN, Zhuo L, Kimata K, Kubes P. Kupffer cells and activation of endothelial TLR4 coordinate neutrophil adhesion within liver sinusoids during endotoxemia. Am J Physiol Gastrointest Liver Physiol (2013) 305(11):G797-806. doi:10.1152/ajpgi.00058.2013

54. McAvoy EF, McDonald B, Parsons SA, Wong CH, Landmann R, Kubes P. The role of CD14 in neutrophil recruitment within the liver microcirculation during endotoxemia. J Immunol (2011) 186(4):2592-601. doi:10.4049/jimmunol. 1002248

Conflict of Interest Statement: The authors declare that the research was conducted in the absence of any commercial or financial relationships that could be construed as a potential conflict of interest.

Received: 31 December 2014; accepted: 02 February 2015; published online: 17 February 2015.

Citation: McDonald B and Kubes P (2015) Interactions between CD44 and hyaluronan in leukocyte trafficking. Front. Immunol. 6:68. doi: 10.3389/fimmu.2015.00068 This article was submitted to Inflammation, a section of the journal Frontiers in Immunology.

Copyright (C) 2015 McDonald and Kubes. This is an open-access article distributed under the terms of the Creative Commons Attribution License (CC BY). The use, distribution or reproduction in other forums is permitted, provided the original author(s) or licensor are credited and that the original publication in this journal is cited, in accordance with accepted academic practice. No use, distribution or reproduction is permitted which does not comply with these terms. 